\title{
Reduction of visual acuity decreases capacity to evaluate radiographic image quality
}

\section{Introduction}

Diagnostic radiographers are required to evaluate radiographs to ensure technical quality. ${ }^{1}$ In some countries formal image interpretation is also provided by radiographers as a diagnostic report. ${ }^{2,3}$ In order to perform these tasks radiographers rely on several attributes: anatomical knowledge, sound radiographic technique, interpretation of image characteristics such as contrast and noise, and the confidence to make informed decisions. ${ }^{4}$

The human visual system (HVS) is crucial to this process, but is not given enough attention as a confounding factor for image evaluation or interpretation. ${ }^{5,6,7}$ The HVS is a sensorial organ composed by the eye, the lateral geniculate nucleus and the visual cortex. These components work together to constantly adapt to different luminance levels and to have a response to frequency that are characteristic to each person since it is involve the eye itself and the brain that processes the information. ${ }^{8,9}$ Previous studies have shown that performance of the eye changes with age, pupil diameter, hyperopia, photopic conditions, and the time of day. ${ }^{10-12}$ Furthermore, only half of the general population is able to identify just noticeable differences (JNDs) due to different levels of contrast sensitivity. ${ }^{7}$ JNDs are considered, in this specific context, the smallest luminance difference that the human observer can detect when an object of a certain size is displayed at a certain background luminance level. ${ }^{13}$

Although most countries will have Health and Safety guidelines for the use of visual display devices, and guidance on ergonomics, there is currently no requirements for eye tests for staff with responsibility for image evaluation and interpretation, there is also no evidence to suggest whether this is necessary. ${ }^{14}$ Therefore, the effect of visual acuity reduction on image evaluation is an important under-researched area. ${ }^{6,12}$

The question of visual acuity testing for radiologists has been raised by several authors in the past ${ }^{15,16}$. The question of radiologists eyesight is clearly related to the detection task and is acknowledged as such in the literature. The fact that fatigue tires the eyes has been extensively studied and is considered and insidious problem, contributing to several catastrophic failures in radiology ${ }^{17-21}$. Visual display devices (VDU) have been linked to fatigue for some time ${ }^{22-26}$ as has surrounding illumination ${ }^{27}$ and viewing distance ${ }^{28}$. VDU syndrome is sometime that Occupational Health practitioners have introduced guidelines. However, when it comes to image quality analysis by radiographers, there have been papers considering radiographers as mammogram reporters ${ }^{29}$, some considering the method and doing preliminary investigation of the literature ${ }^{30,31}$ which concluded "Further studies are necessary to understand fully the relationship between visual performance on tests and diagnostic accuracy in practice". Accordingly, this study has the following objectives: a) to determine the impact of reduced visual acuity on a simple task (object counting in a phantom); and b) assess the impact of reduced visual acuity on the technical evaluation of appendicular radiographs. 


\section{Methods}

Ethical approval was granted by the Research Ethics Panel, School of Health and Society, University of Salford (HSR1819-115). Written informed consent was obtained from each observer prior to participation in the study. Information was provided to observers using a poster and an information sheet. Observers could withdraw at any time and no incentives were offered.

\section{Observers}

Participants were BSc Radiography students from 5 institutions from across Europe and South Africa who were attending the OPTIMAX summer school. Observers wearing prescription glasses were required to use them when performing the experimental tasks and 'simulation glasses ${ }^{29}$ were placed on top of them. The same simulation glasses were also used for observers who did not wear prescription glasses.

\section{Viewing Conditions/Materials}

A 2.3 Megapixel NEC EA243WM (Tokyo, Japan) monitor was used in this study, exceeding the minimum recommended specification for clinical review set out in The Royal College of Radiologists Picture archiving and communication systems (PACS) and guidelines on diagnostic display device. ${ }^{32}$ The monitors were calibrated to the American College of Radiology (ACR) and the National Electrical Manufacturers Association (NEMA) (ACR-NEMA) Digital Imaging and Communications in Medicine (DICOM) part 14 standard $^{33}$ using a Society of Motion Picture and Television Engineers (SMPTE) RP133 test pattern. ${ }^{34}$ Ambient lighting was controlled using an X2 RaySafe lux meter (Billdal, Sweden) and maintained at 7 lux (standard error \pm 1 lux) as suggested in the literature and by the European Commission. ${ }^{35-39}$ Observers were positioned at a distance of $60 \mathrm{~cm}$ from the monitor for both phases of this study. ${ }^{32}$

\section{Simulation Glasses}

To achieve a reduction in visual acuity, simulation glasses from the University of Cambridge inclusivity toolkit (Cambridge, UK) were used. The visual impact of the glasses has been tested previously ${ }^{40-42}$, using the Logarithm of the Minimum Angle of Resolution (log MAR) as a scale to score the visual acuity. One pair of glasses results in a reduction of $0.08 \log M A R$, while 2 pairs gives reduction of $0.26 \mathrm{log} \operatorname{MAR}^{43}$ (Figure 1). These equate roughly to 20/25 and 20/40 vision on the imperial Snellen scale ${ }^{43}$, respectively. One pair of glasses was used to degrade slightly the vision and 2 pairs were used to degrade it further to identify the impact of visual degradation on the identification of subtle anatomical details.

(a) (b) (c) 

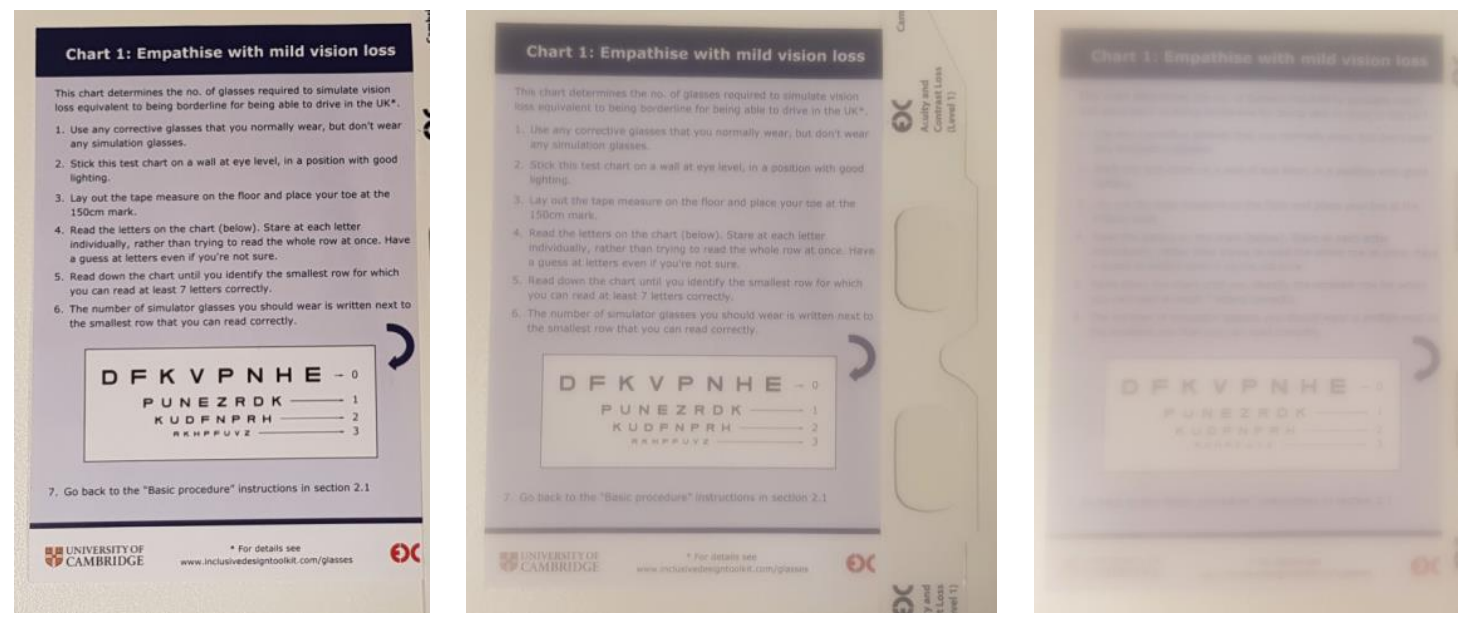

Figure 1: Cambridge Simulation Loss of Contrast Glasses - impact on the visualisation of text: (a) no glasses, (b) uses of one pair of glasses - shown aside, and (c) two pairs of glasses.

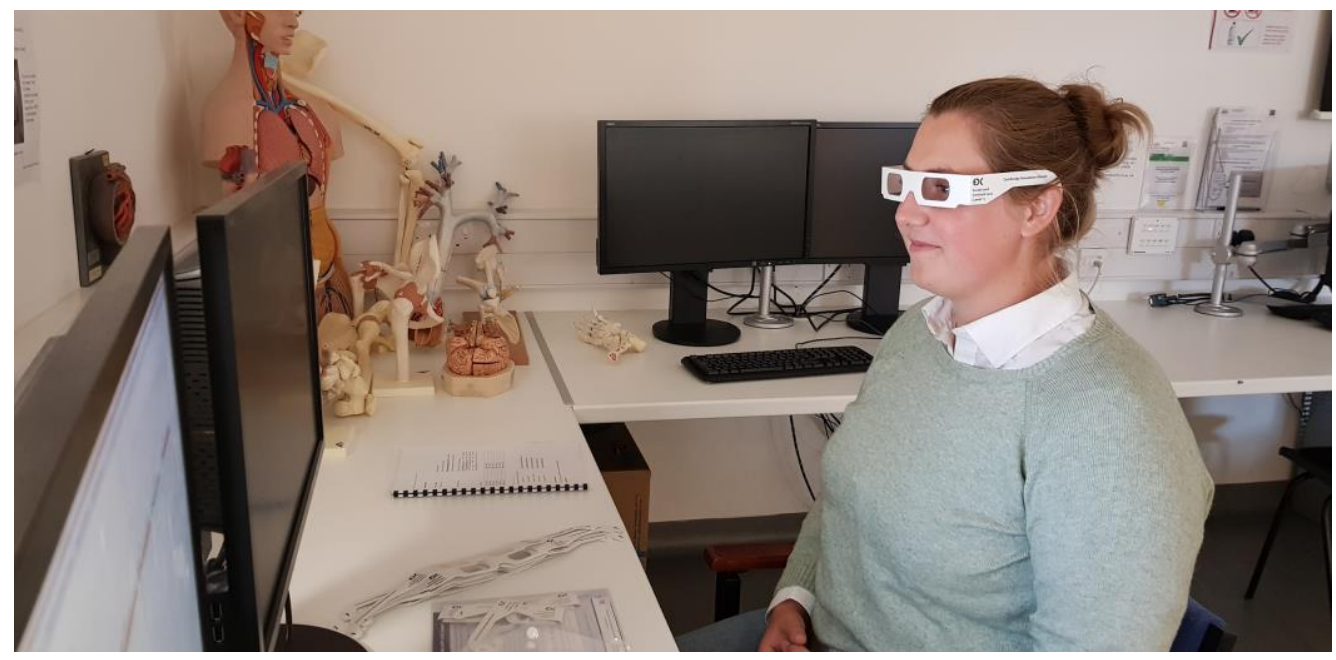

Figure 2: Demonstration of Cambridge Simulation Glasses in use.

\section{Phase 1: Phantom Study}

Two types of phantom images were used for phase 1 of this study: the TORMAM phantom (Figure 3) and the RaySafe Pro-Digi Radiography phantom (Billdal, Sweden) (Figure 4). RaySafe Pro-Digi Radiography is a multi-functional phantom designed to analyse beam alignment, dynamic range, contrast resolution, spatial resolution, and homogeneity of image having different tools to verify all these parameters. Images of the TORMAM phantom included 6 groups of multi-directional filaments, 6 groups of microcalcifications and 6 groups of 3 nodules with different contrast levels. ${ }^{44,45}$ Phantom images are homogeneous and represent a "signalknown-exactly/background-known-exactly" ("SKE/BKE") test. This can help predict the outcome in clinical images. Observers received training to evaluate the phantoms, which comprised counting objects of each type within the image.

All phantom images were displayed using ImageJ software (Maryland, USA). Using the phantom images, the visual task was undertaken in three conditions. First with 2 pairs of glasses simulation glasses, second with 1 pair of glasses, third without simulation glasses. After each visual task, observers had a break of 30 to 45 minutes to minimize the impact of fatigue. 

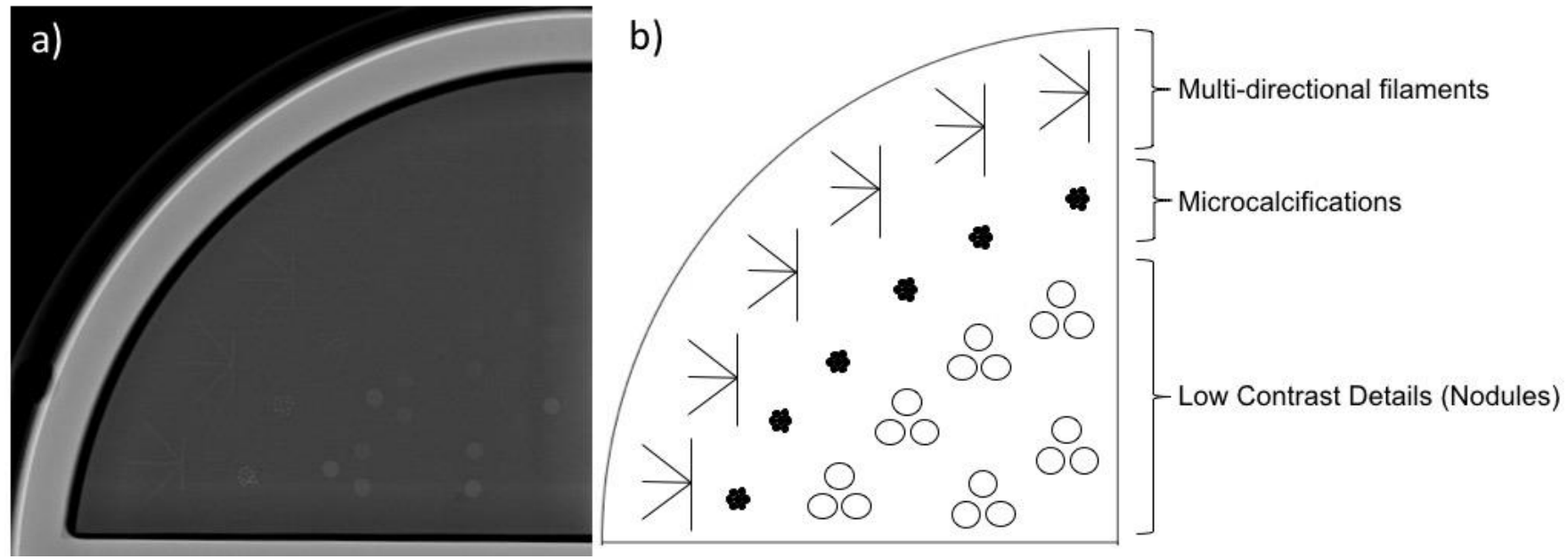

Figure 3 - TORMAM phantom: a) clinical image of the phantom and b) scheme of the details observed (multi-directional filaments, microcalcifications and low contrast details simulating nodules. For each detail under assessment, the observers needed to attribute 3 if it was clearly visible and well defined, 2 was attributed when was visible, 1 if it was not defined but still visible and " 0 " if it was not visible at all. ${ }^{32}$

(a)

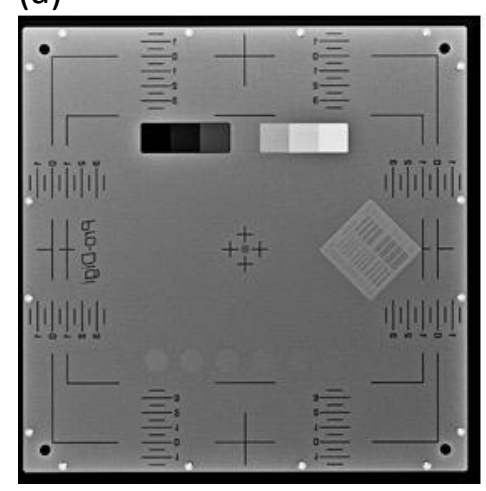

(b)

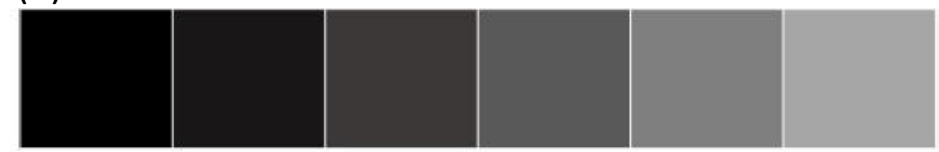

\section{Grey Scales (Squares and Circles)}

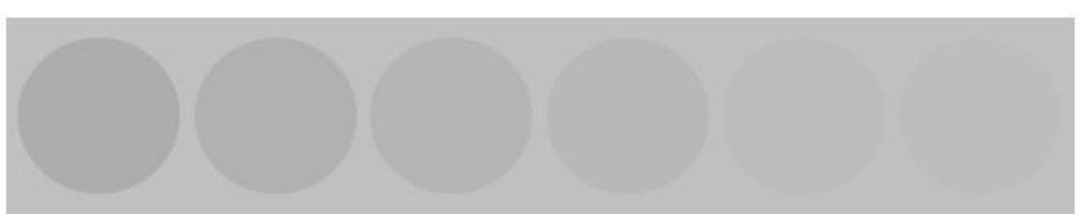

Figure 4 - RaySafe Pro-Digi Radiography phantom image (a) and the details that observers needed to evaluate by counting (b).

\section{Phase 2: Clinical Image Evaluation Study}

Clinical images were also used to see the impact of anatomical variation and differences in contrast and brightness of structures on image evaluation with reduced visual acuity. The patient variations can promote image quality variations making comparisons more difficult. For that, thirty anonymized appendicular skeletal radiographic images were selected: hands $(n=6)$, tibia/fibula $(n=5)$, shoulder $(n=1)$, wrist $(n=6)$, foot $(n=6)$, ankle $(n=4)$, knees $(n=2)$. Observers had an explanation of how to evaluate image quality and use the visual grading scale in ViewDEX. ${ }^{46,47}$

ViewDex (v.2.0) software (Brämhultsvägen, Sweden) was utilized to display images and capture observer responses. ${ }^{46,47}$ The criteria from the DIMOND $3^{48}$ report were used as reference to develop the five questions (Figure 5) used to perform image evaluation. 
Additionally, questions were also designed to include broader anatomical aspects (soft tissues, cortical and trabecular bone) to be applicable to all of the radiographic images, regardless of body part. A 4-point Likert scale was used to identify the definition of anatomical structures. ${ }^{49}$ During the second phase, 7 out of 13 observers participated.

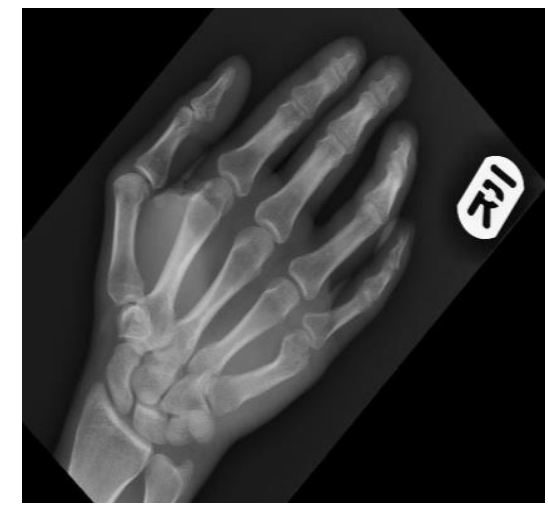

\begin{tabular}{|c|c|c|c|}
\hline \multicolumn{4}{|c|}{ The cortical margins of the bone are } \\
\hline $1-$ Not Defined & 2 - Slightly defined & 3 - Defined & 4-Clearly defined \\
\hline \multicolumn{4}{|c|}{ The trabecular patterns of the bone are } \\
\hline $1-$ Not Defined & 2 - Slightly defined & 3- Defined & 4-Clearly defined \\
\hline \multicolumn{4}{|c|}{ The joint spaces between the bones are } \\
\hline $1-$ Not Defined & 2 - Slightly defined & 3- Defined & 4-Clearly defined \\
\hline \multicolumn{4}{|c|}{ The skin borders (in the entire image) are } \\
\hline $1-$ Not Defined & 2 - Slightly defined & 3- Defined & 4-Clearly defined \\
\hline \multicolumn{4}{|c|}{ The contrast between fat and muscle is } \\
\hline $1-$ Not Defined & 2 - Slightly defined & 3 - Defined & 4-Clearly defined \\
\hline
\end{tabular}

Figure 5: Example of a clinical image assessed by the observers, the respective questions and the rating scale varying from 1 (not defined) to 4 (clearly defined) that were presented to the observers using ViewDEX.

\section{Data Analysis}

Descriptive statistics were managed using MS Excel and IBM SPSS statistics version 25 . For the phantom study, descriptive statistics were used, and a Friedman test was performed. For the clinical image evaluation, visual grading characteristic (VGC) analysis was performed.

Inferential statistics were carried out using SPSS and VGC analysis using VGC Analyzer version 1.0.2. ${ }^{50}$ VGC analysis is a non-parametric and rank-invariant method for analysing visual grading data. It produces a VGC curve in which the image quality ratings for two different conditions [reference condition (no glasses) and a test condition (wearing glasses)] were compared one against the other by Area Under the VGC Curve ( $A \cup C_{V G C}$ ) plotting the VGA data of one against the other. An $A_{U} C_{V G C}$ of 0.5 suggests that the two conditions being compared are providing similar fulfilment of image quality criteria, but if the $95 \%$ confidence interval of AUC $_{V G C}$ does not include 0.5 , then the conditions are considered statistically different. In this test an AUC below 0.5 suggest preference for wearing no glasses. VGC Analyzer is a valid option ${ }^{51}$ for the quantitative assessment of image quality and has many advantages including high validity ${ }^{52}$ and ViewDEX has similar functionality to the viewing software available in clinical practice. ${ }^{53}$

\section{Results}

The observers participating in both phases had an average age of $21.9 \pm 2.75$ years old (Table 1 ). The majority of the observers (7/13) had a recent eye test $(<1$ year), two had their last vision checked in the past two years and the remaining, more than 3 years ago and 8/13 observers wore corrective lenses (Table 1). 
Table 1 - Demographic data of observers, visual acuity and the use of correction if needed.

\begin{tabular}{cccccc}
\hline Observers & Age & Gender & Year of study (BSc) & Last Eye Test & Use of Correction \\
\hline Obs1 & 19 & $\mathrm{~F}$ & 2 & $<2$ years & No \\
Obs2 & 21 & $\mathrm{~F}$ & 2 & $<1$ year & Yes \\
Obs3 & 24 & $\mathrm{M}$ & 2 & $>3$ years & Yes \\
Obs4 & 24 & $\mathrm{M}$ & 2 & $<2$ years & Yes \\
Obs5 & 21 & $\mathrm{~F}$ & 2 & $<1$ year & No \\
Obs6 & 21 & $\mathrm{~F}$ & 2 & $>3$ years & Yes \\
Obs7 & 23 & $\mathrm{M}$ & 3 & $>3$ years & No \\
Obs8 & 21 & $\mathrm{~F}$ & 3 & $<1$ year & No \\
Obs9 & 21 & $\mathrm{~F}$ & 2 & $<1$ year & Yes \\
Obs10 & 19 & $\mathrm{~F}$ & 1 & $<1$ year & Yes \\
Obs11 & 29 & $\mathrm{~F}$ & 4 & $>3$ years & No \\
Obs12 & 19 & $\mathrm{M}$ & 1 & $<1$ year & Yes \\
Obs13 & 23 & $\mathrm{~F}$ & 2 & $<1$ year & Yes \\
\hline
\end{tabular}

\section{Phase 1: Phantom Study}

Phantom images were assessed by 13 BSc radiography students. With 2 pairs of glasses the mean number of details identified was $9.86 \pm 1.03$ (out of 11), for one pair of glasses was 9.69 \pm 1.11 and without glasses was $9.92 \pm 1.89$ (Figure 6a). Therefore, the grey-scale image did not differentiate performance with and without glasses $(p=0.325)$ and the mean values are very similar (the mean difference was 0.23 with or without glasses, and no difference between 1 or 2 glasses). No significant statistical difference was identified using Friedman test $(p=0.325)$.

(a)

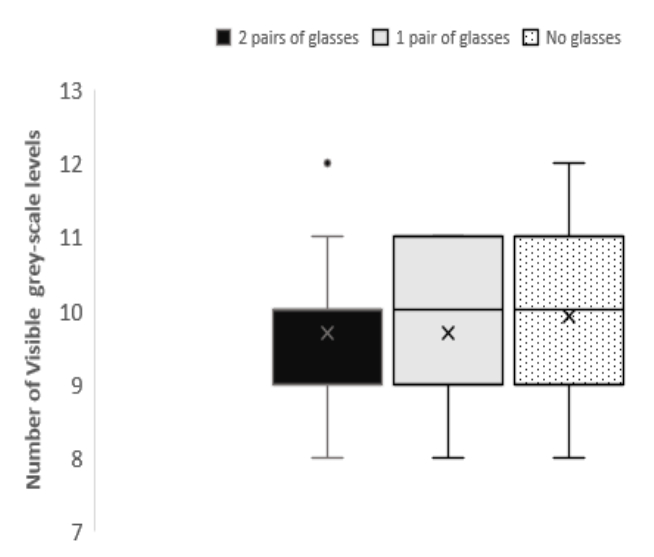

(b)

m 2 pairs of glasses $\square 1$ pair of glasses $\square$ No glasses

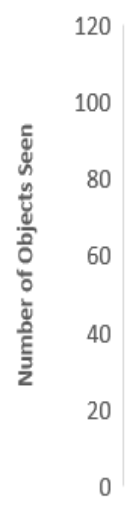

Figure 6 - Number of grey levels visible on RaySafe Pro-Digi phantom images: the box plot shows the mean $(x)$, the median, interquartile range and outliers $(\bullet)$. The handles in boxplot represent minimum and the maximum. No outliers observed. (a) grayscale counting, (b) TOR MAN images.

The objects on TOR MAM images were hardly visible when using a 2 pairs of simulation glasses (Figure 6b), as demonstrated by a mean value of 14.62 varying between 7 and 31 . For one pair of simulation glasses the mean was 54.62 (14 - 95) and without glasses, the mean was 86.92 
(49 - 109). The use of 2 and 1 pairs of glasses promoted statistically significant differences in the detection of TOR MAM details $(p=0.001)$ using the Friedman test when observers assessed TOR MAM images (Figure 6).

It was noticed that the mean time spend by the observers to count the details varied. Time was shorter when observers were with 2 pairs of glasses $(X=3.0 \mathrm{~min}$ ) and a little longer $(X=3.5$ $\mathrm{min}$ ) when they were wearing 1 pair of glasses (Figure 9).

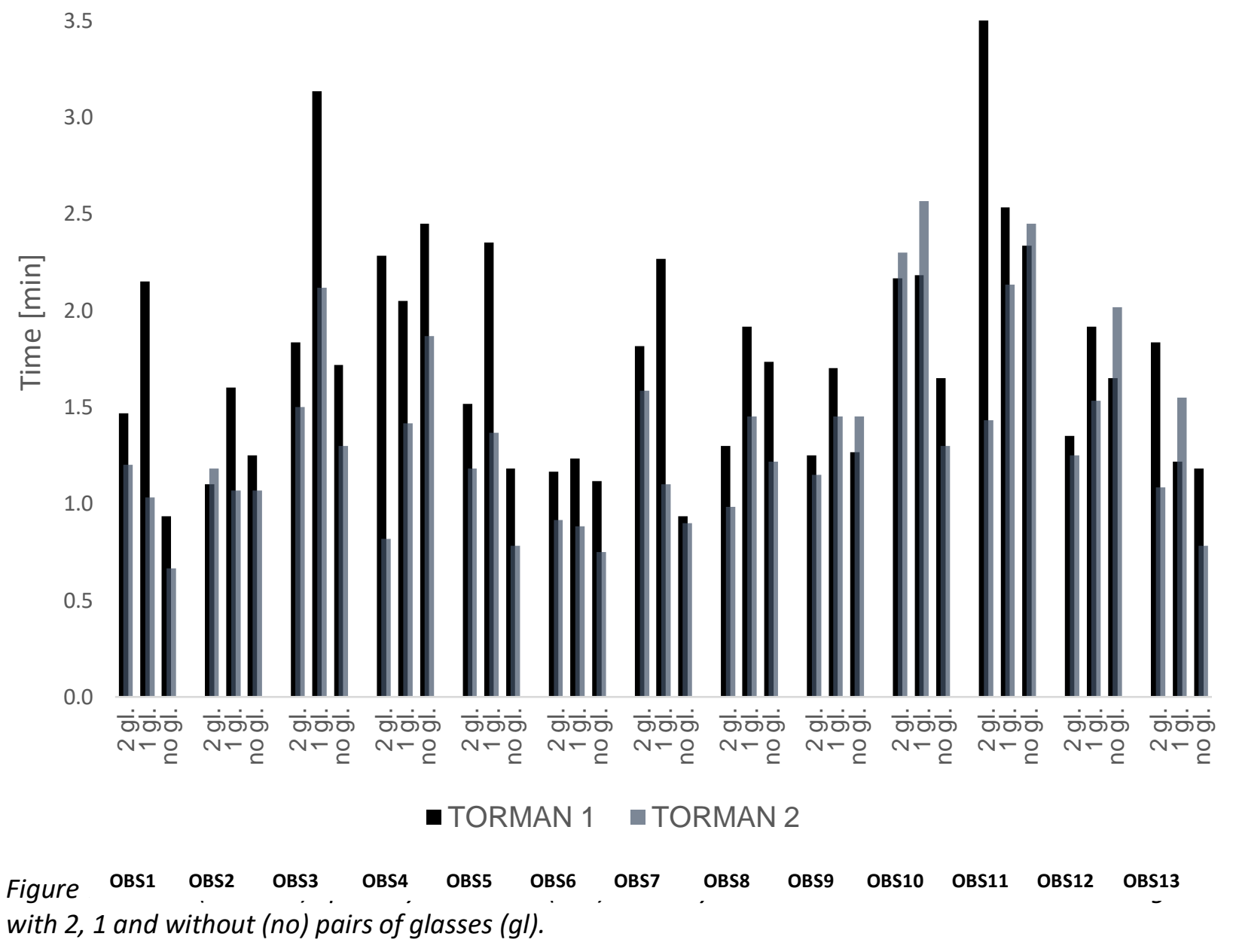

\section{Phase 2: Clinical Image Evaluation}

For the second phase, 7 observers completed the evaluation of clinical images. Six observers had corrected vision using glasses or contact lenses, while one did not. Visual performance was better when no simulation glasses were used, compared with one or two pairs of simulation glasses (Figure 8, Table 2). The $\mathrm{AUC}_{\mathrm{VGC}}$ showed that observers can lose anatomical information and detect fewer contrast differences when they have reduced visual acuity, imposed by the simulation glasses (see Table 2). 


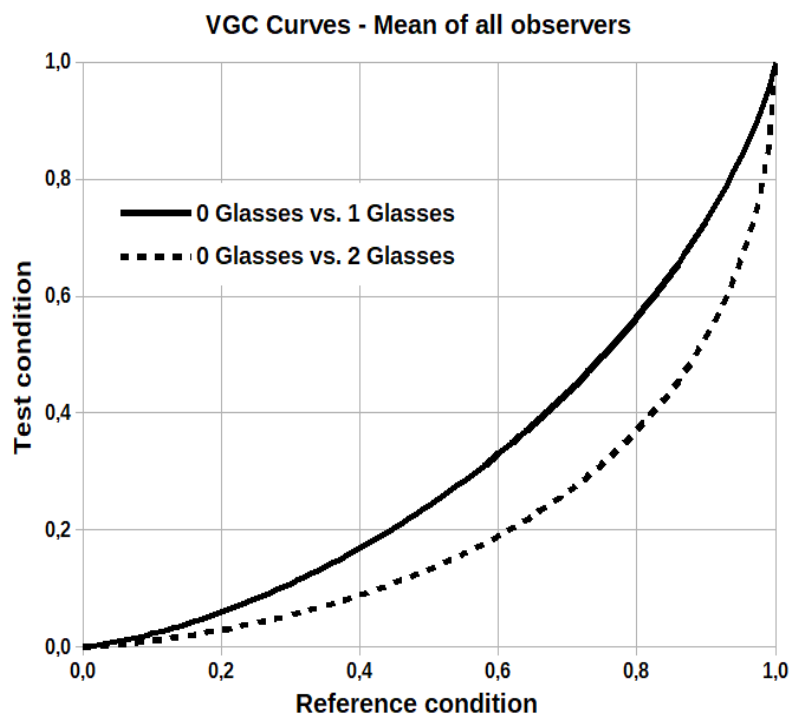

Figure 8 - Mean values of VGC curves generated to compare the observers not wearing glasses (Reference Condition) versus 1 and 2 pairs of glasses (Test Condition).

Table 2 - Area under the curve $(A \cup C)$ - binomial approximation.

\begin{tabular}{l|c|c}
\hline \multicolumn{1}{c|}{ VCG Curves } & 0 Glasses vs 1 Glasses & 0 Glasses vs 2 Glasses \\
\hline Area \& & 0.303 & 0.197 \\
95\% confidence interval & $(0.280,0.333)$ & $(0.175,0.223)$ \\
\hline Standard Deviation & 0.014 & 0.012 \\
\hline
\end{tabular}

Comparing the challenge of anatomical image evaluation when observers were wearing 1 pair of simulation glasses to 0 pairs, it was possible to verify that the skin borders in the images were the most difficult anatomical area to evaluate. On the other hand, the evaluation of joints spaces did not differ when observers changed from 2 to 1 pairs of glasses (Figure 9a \& 9b). The differentiation between trabecular and cortical bone, fat and muscle soft tissues was better when observers had no glasses (Figure 9a).

(a)

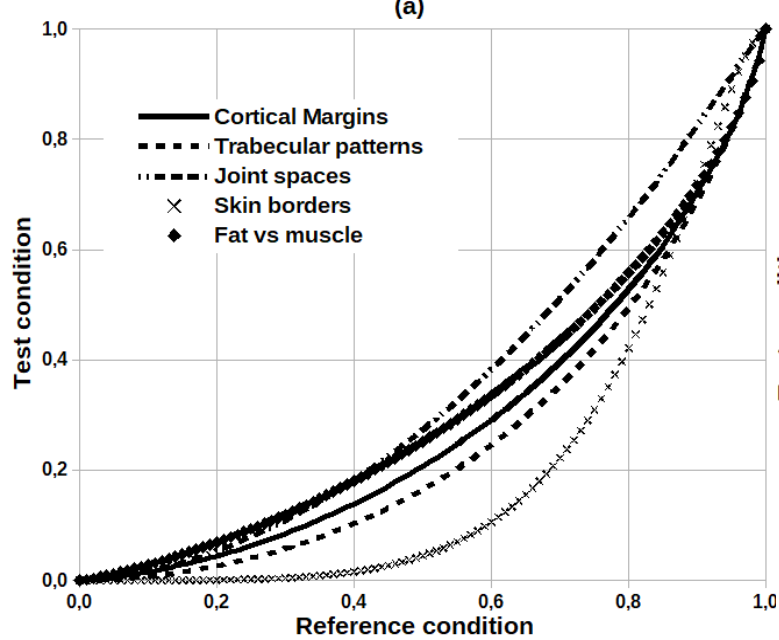

(b)

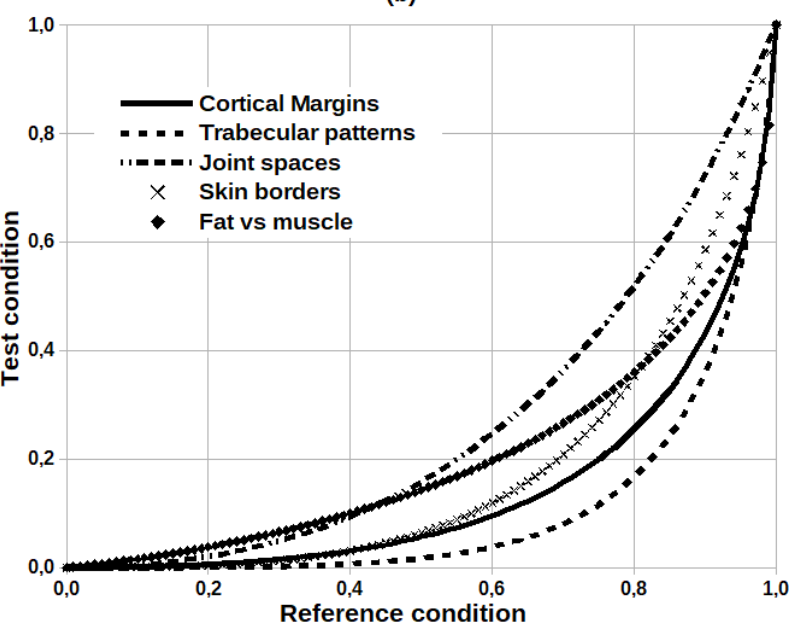

Figure 9 - VGC curves calculated for each individual answer related to each anatomical detail (trabecular and cortical bone, joint spaces, skin, fat and muscle) evaluated by the observers with: (a) 1 
pairs glasses as test condition, (b) 2 pairs glasses as test condition. Reference condition is considered as no simulation glasses on.

When the observers' performance to evaluate anatomical details was compared between 2 pairs of simulation glasses and no glasses (reference condition), it was possible to verify the same tendency. The joint spaces were less challenging to identify, while the trabecular pattern and cortical margins of the bone were harder to identify. The skin edges, muscles and fat differentiation were more difficult to detect when 2 pairs of simulation glasses were used (Figure 9b).

For the clinical images, the observers needed less time when they did not use simulation glasses $(8.9 \pm 2.2 \mathrm{~min})$, compared to when they had 1 pair of simulation glasses $(12.1 \pm 3.3$ $\mathrm{min})$ and 2 pairs of simulation glasses $(15.4 \pm 3.6 \mathrm{~min})$. Wearing 1 pair of simulation glasses promoted an increase of about $36 \%$ of time (minutes), while 2 pairs promoted an increase of $72 \%$ (Figure 10).

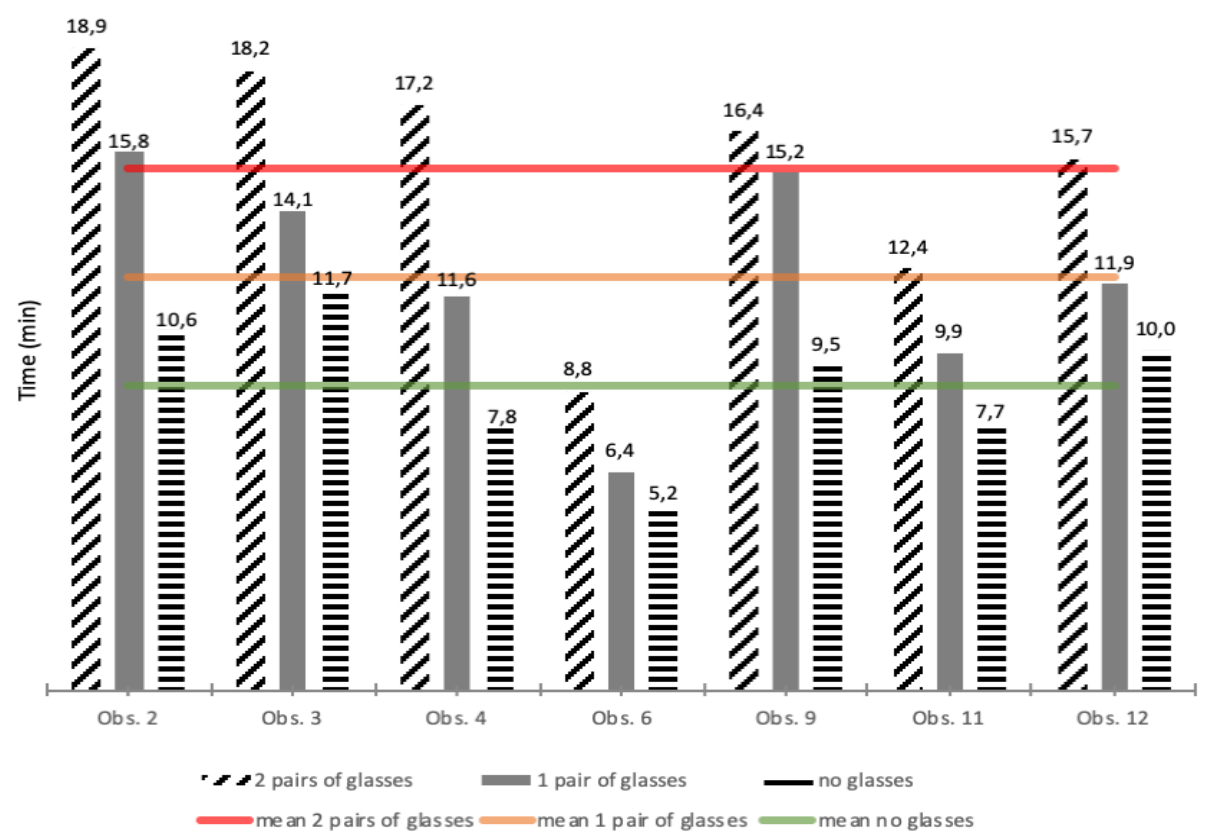

Figure 10 - Time spent by each observer with 2 pair of glasses, 1 pair of glasses and no glasses, with the mean values for each condition.

\section{Discussion}

The results confirm that a reduction in visual acuity can negatively impact object counting exercises and the evaluation of image quality in clinical images. Not only was the counting and the identification of details affected but also the time required to perform these tasks increased with reduced visual acuity. This may indicate that radiographers' image quality evaluation can be impacted by visual acuity. Radiographers are the first health professionals to see a patients' radiographic images, they make decision based on image acquisition, postprocessing and evaluation. $2,3,54,55$ If their visual acuity is compromised this could negatively affect in clinical image evaluation. Low contrast and smaller size objects were more challenging to identify when the observers were using 2 pairs of glasses (Figure 6). The Interquartile Range (IQR) for images assessed with no glasses was 60, showing an increase on 
the number of details identified. The time spent performing this task varied, being lower for high contrast details when observers were wearing 2 pairs of glasses and longer for smaller low contrast details. The shorter time associated with wearing 2 pairs of glasses could be due to the inability to distinguish the objects because of the visual acuity reduction, thus forcing the observers move to the next stage of the task. For the images with low contrast details no difference was noted in the evaluation, meaning that the performance was similar (99.86, $9.69,9.92$ ) when the observers were with or without glasses (Figure 6). This result may be explained by the contrast sensitivity of each individual observer which can vary ${ }^{10,12}$ meaning details are visible for some and not visible for others.

Observer performance deteriorated with visual acuity reduction in the identification of the specific anatomical details that were evaluated on clinical images. The negative impact was observed in the detection of anatomical details and also on the longer time spent performing the task. With 1 pair of glasses, the visualisation of the soft tissues was the more difficult anatomical detail to identify. Soft tissue details are considered important because it is an example of low contrast detail, it also is an indicator of the margins of the image. An inability to see it this details may make it impossible to make a decision as to whether the correct anatomical area has been included. A further reduction (2 pairs of glasses) in visual acuity reduced the visualisation of trabecular patterns and cortical margins, both of which are also important to consider in image quality evaluation ${ }^{48}$ mainly when looking for fractures. As observed in the details counting, the anatomical structures that have higher contrast (joint spaces) were visualised more clearly compared to those with low contrast as soft tissues.

The image evaluation time was faster without glasses, this may be due to normal visual acuity, but it can also be due to the strategy of putting observers analysing images using 2 pairs of glasses, then with 1 pair of glasses, and finally without glasses. The selected method may have made them more familiar with the images and software manipulation, which will make them work faster with time.

The Health and Care Professions Council $(\mathrm{HCPC})^{54}$ states that radiographers need to: "be able to maintain fitness to practice and be able to assure the quality of their practice", this clearly includes the ability to see the images they produce. It is self-evident that if a radiographer cannot adequately evaluate the diagnostic quality of the image; where they have reduced visual performance, they are more likely to make inappropriate decisions on image quality. Errors would be more likely to occur where low contrast detail or high noise is at question. Digital radiography systems can compensate for under and over exposure but where noise is high, the digital system will adjust the brightness, and may reduce noise, masking the lack of anatomical details. This type of difference is subtle and is likely to be made more difficult by a lack of visual acuity. Further work is needed to understand the thresholds of visibility.

The main contribution of this study to the radiographers' clinical practice is related to the opportunity to analyse and discuss the impact of visual performance on daily tasks, that may affect examination outcomes and potentially patient management. Regarding the published literature, the eye performance is overlooked and there is no evidence that training, viewing conditions or experience can overcome the visual acuity reduction. ${ }^{14,29,35,37,56,57}$ From the results obtained, it seems that visual performance needs to be considered to reduce the risks associated with incomplete or incorrect diagnosis. If employers or professional bodies were to introduce regular eye tests into health screening it may reduce the risk of misinterpretation 
as a result of poor vision. The eye screening tests, and correction measures may assist in improving radiology practice service standards and further enhancing quality assurance. This could help to ensure that any changes in visual acuity are corrected accordingly. These proposed refining methods could safeguard image evaluation and review from being impeded by visual acuity reduction, as number of professions whose expertise affect the safety of the general public have a basis of visual standard. ${ }^{5}$

There are some limitations to the current work. There was no optometrist available to measure and correct the differences on observers' vision acuity before data collection, this would have helped to establish a baseline of visual acuity for each observer. This study only included observers with short experience in radiography and it would be interesting to include more aged to better represent radiographers' population and also more experienced to see if experience would overcome eventual visual acuity reduction. According to the literature image evaluation can be affected by the level of experience/expertise. ${ }^{56}$ As the level of expertise increases, the ability to evaluate images accurately improves. This means that even with compromised vision, the professional would be able to evaluate images up to the standard requirements owing the adaptive capabilities of the eyes. The counting of objects and image evaluation were carried out on calibrated 2.3 MP resolution monitor that would be classed as computers off the shelf (COTS). These are similar in specification to those used in clinical practice by the radiographers for initial assessment. However, that can be seen as a limitation because in previous work it was shown that this type of monitors does not keep their calibration compared to medical grade monitors used for primary diagnosis. ${ }^{58}$

\section{Conclusion}

A visual acuity reduction negatively affects image quality evaluation, therefore, radiographers with visual acuity reduction may be more prone to incorrectly appraising the quality of images due to the lack of identification of artefacts or other low contrast details.

\section{References}

1. Field LJ., Snaith BA. Developing radiographer roles in the context of advanced and consultant practice. $J$ Med Radiat Sci 2013;60(1):11-5. Doi: 10.1002/jmrs.2.

2. Hardy M., Snaith B. Radiographer interpretation of trauma radiographs: Issues for radiography education providers. Radiography 2009;15(2):101-5. Doi: 10.1016/j.radi.2007.10.004.

3. Snaith B., Hardy M., Lewis EF. Radiographer reporting in the UK: A longitudinal analysis. Radiography 2015;21(2):119-23. Doi: 10.1016/j.radi.2014.10.001.

4. Donovan T., Manning DJ. Successful reporting by non-medical practitioners such as radiographers, will always be task-specific and limited in scope. Radiography 2006;12(1):7-12. Doi: 10.1016/j.radi.2005.01.004.

5. Wong NWK., Stokes J., Foss AJE., McGraw P V. Should there be a visual standard for ophthalmologists and other surgeons? Postgrad Med J 2010;86(1016):354-8. Doi: 10.1136/pgmj.2009.091371.

6. Safdar NM., Siddiqui KM., Qureshi F., Mirza MK., Knight N., Nagy P., et al. Vision and Quality in the Digital Imaging Environment: How Much Does the Visual Acuity of Radiologists Vary at an Intermediate Distance? Am J Roentgenol 2009;192(6):W335-40. Doi: 10.2214/AJR.07.3515.

7. Barten PGJ. Physical model for the contrast sensitivity of the human eye. In: Rogowitz BE, editor. Proceedings of the SPIE, 1992, p. 57.

8. Rizzi A., Bonanomi C. The human visual system described through visual illusions. Colour Design, Elsevier; 2017, p. 23-41.

9. Ji T-L., Sundareshan MK., Roehrig H. Adaptive image contrast enhancement based on human visual properties. IEEE Trans Med Imaging 1994;13(4):573-86. Doi: 10.1109/42.363111. 
10. Barten PGJ. Contrast Sensitivity of the Human Eye and Its Effects on Image Quality, 1st Editio, Technische Universiteit Eindhoven; 1999.

11. Derefeldt G., Lennerstrand G., Lundh B. Age Variations in Normal Human Contrast Sensivity. Acta Ophthalmol 2009;57(4):679-90. Doi: 10.1111/j.1755-3768.1979.tb00517.x.

12. Seyhan Karatepe A., Köse S., Eğrilmez S. Factors Affecting Contrast Sensitivity in Healthy Individuals: A Pilot Study. Türk Oftalmol Derg 2017;47(2):80-4. Doi: 10.4274/tjo.93763.

13. Ji TL., Sunderaeshan MK., Roehrig H. Adaptative Image Contrast Enhancement Based on Human Visual Properties. IEEE Trans Med Imaging 1994;13(4):573-86.

14. Krupinski EA., Berbaum KS., Caldwell R. Impact of visual fatigue on observer performance. In: Sahiner B, and Manning DJ, editors. Medical Imaging 2009: Image Perception, Observer Performance, and Technology Assessment, 2009, p. 726310.

15. Quaghebeur G., Bhattacharya JJ., Murfitt J. Radiologists and visual acuity. Eur Radiol 1997;7(1):41-3. Doi: 10.1007/s003300050106.

16. Straub WH., Gur D., Good BC. Visual acuity testing of radiologists - is it time? Am J Roentgenol 1991:11078. Doi: 10.2214/ajr.156.5.2017936.

17. Krupinski EA., Berbaum KS., Caldwell R. Impact of visual fatigue on observer performance. In: Sahiner B, and Manning DJ, editors. Medical Imaging 2009: Image Perception, Observer Performance, and Technology Assessment, 2009, p. 726310.

18. Reiner BI., Krupinski E. Demystifying occupational stress and fatigue through the creation of an adaptive end-user profiling system. J Digit Imaging 2012;25(2):201-5. Doi: 10.1007/s10278-011-9441-7.

19. Krupinski EA. Reader fatigue interpreting mammograms. Lecture Notes in Computer Science (including subseries Lecture Notes in Artificial Intelligence and Lecture Notes in Bioinformatics), vol. 6136 LNCS, 2010, p. 312-8.

20. Krupinski E., Reiner BI. Real-time occupational stress and fatigue measurement in medical imaging practice. J Digit Imaging 2012;25(3):319-24. Doi: 10.1007/s10278-011-9439-1.

21. Stec N., Arje D., Moody AR., Krupinski EA., Tyrrell PN. A systematic review of fatigue in radiology: Is it a problem? Am J Roentgenol 2018:799-806. Doi: 10.2214/AJR.17.18613.

22. Displays NO-., 1985 undefined. The effect of VDU colour on visual fatigue in the fovea and periphery of the visual field. Elsevier n.d.

23. Padmos P. Visual Fatigue with Work on Visual Display Units: The Current State of Knowledge. HumanComputer Interaction, Springer Berlin Heidelberg; 1988, p. 41-52.

24. Dillon T., Behavior HE-C in H., 1996 undefined. Some factors affecting reports of visual fatigue resulting from use of a VDU. Elsevier n.d.

25. Gur S., Ron S., Heicklen-Klein A. Objective evaluation of visual fatigue in VDU workers, vol. 44, 1994.

26. Watten RG., Lie I., Magnussen S. VDU work, contrast adaptation, and visual fatigue. Behav Inf Technol 1992;11(5):256-61. Doi: 10.1080/01449299208924347.

27. Bullough J., Akashi Y., ... CF-J of A., 2006 undefined. Impact of surrounding illumination on visual fatigue and eyestrain while viewing television. DocsdriveCom n.d.

28. Jaschtnski-Kruza W. Visual strain during VDU work: The effect of viewing distance and dark focus. Ergonomics 1988;31(10):1449-65. Doi: 10.1080/00140138808966788.

29. Thompson J., Denton E., Lança C., Lança L., Hogg P. The impact of reduced visual acuity and fatigue on lesion detection in mammography 2019. Doi: 10.26044/ecr2019/C-1364.

30. Thompson JD., Lança C., Lança L., Hogg P. A method to determine the impact of reduced visual function on nodule detection performance. Radiography 2017;23(1):19-24. Doi: 10.1016/j.radi.2016.07.002.

31. Lança C., Thompson JD., Lança L., Hogg P. Visual Function Assessment in Medical Imaging Research. Radiol Technol n.d.;87(2):129-38.

32. The Royal College Of Radiologists. Picture archiving and communication systems (PACS) and guidelines on diagnostic display devices, 2019.

33. Mcllgorm DJ., McNulty JP. DICOM part 14: GSDF-calibrated medical grade monitor vs a DICOM part 14: GSDF-calibrated "commercial off-the-shelf" (COTS) monitor for viewing 8-bit dental images. Dentomaxillofacial Radiol 2015;44(3):20140148. Doi: 10.1259/dmfr.20140148.

34. Gray JE. Use of the SMPTE test pattern in picture archiving and communication systems. J Digit Imaging 1992;5(1):54-8. Doi: 10.1007/BF03167824.

35. Lança C., Thompson JD., Lança L., Hogg P. Visual Function Assessment in Medical Imaging Research. Radiol Technol n.d.;87(2):129-38.

36. Kumar R. Research Methodology: A Step-By-Step Guide for Beginners, 3rd Editio, London: SAGE Publications Ltd; 2011. 
37. Brennan PC., McEntee M., Evanoff M., Phillips P., O'Connor WT., Manning DJ. Ambient Lighting: Effect of Illumination on Soft-Copy Viewing of Radiographs of the Wrist. Am J Roentgenol 2007;188(2):W177-80. Doi: 10.2214/AJR.05.2048.

38. Pollard BJ., Samei E., Chawla AS., Beam C., Heyneman LE., Koweek LMH., et al. The Effects of Ambient Lighting in Chest Radiology Reading Rooms. J Digit Imaging 2012;25(4):520-6. Doi: 10.1007/s10278-0129459-5.

39. European Comission. Criteria for acceptability of radiological (including radiotherapy) and nuclear medicine installations 1997:1-26.

40. Goodman-Deane J., Waller S., Collins A-C., Clarkson., John P. Simulating Vision Loss. Contemporary Ergonomics and Human Factors, ROUTLEDGE in association with GSE Research; 2013, p. 347-354(8).

41. Allen PM., Latham K., Mann DL., Ravensbergen RHJC., Myint J. The Level of Vision Necessary for Competitive Performance in Rifle Shooting: Setting the Standards for Paralympic Shooting with Vision Impairment. Front Psychol 2016;7. Doi: 10.3389/fpsyg.2016.01731.

42. Rae S., Latham K., Katsou MF. Meeting the UK driving vision standards with reduced contrast sensitivity. Eye 2016;30(1):89-94. Doi: 10.1038/eye.2015.188.

43. Snellen L. Conversion table, 2015.

44. Leeds. Leeds test objects. TOR MAs TOR MAX 2011:1-3.

45. Sá dos Reis C. Equipment Quality Control. In: Hogg P, Kelly J, and Mercer C, editors. Digital Mammography - A holistic approach, Springer; 2015, p. 143-52.

46. Håkansson M., Svensson S., Zachrisson S., Svalkvist A., Båth M., Månsson LG. ViewDEX 2.0: a Java-based DICOM-compatible software for observer performance studies. In: Sahiner B, and Manning DJ, editors. Medical Imaging 2009: Image Perception, Observer Performance, and Technology Assessment 2009 Mar 12 (Vol. 7263, p. 72631G)., 2009, p. 72631G.

47. Hakansson M., Svensson S., Zachrisson S., Svalkvist A., Bath M., Mansson LG. VIEWDEX: an efficient and easy-to-use software for observer performance studies. Radiat Prot Dosimetry 2010;139(1-3):42-51. Doi: $10.1093 / \mathrm{rpd} / \mathrm{ncq} 057$.

48. Busch H., Busch Chr Decker Dipl Ing Schilz Dipl Ing A Jockenhöfer St Busch candmed M Anschütz MTRA Lead Contractor HC. DIMOND III Image Quality and Dose Management For Digital Radiography Final Report DIMOND III Working Group: Brüderkrankenhaus Trier Germany, 2004.

49. Shiner N., Howard ML. The use of simulation and moulage in undergraduate diagnostic radiography education: A burns scenario. Radiography 2019;25(3):194-201. Doi: 10.1016/j.radi.2018.12.015.

50. Båth M., Hansson J. Vgc Analyzer: A Software For Statistical Analysis Of Fully Crossed Multiple-Reader Multiple-Case Visual Grading Characteristics Studies. Radiat Prot Dosimetry 2016;169(1-4):46-53. Doi: $10.1093 / r p d / n c v 542$.

51. Hansson J., Månsson LG., Båth M. The Validity Of Using Roc Software For Analysing Visual Grading Characteristics Data: An Investigation Based On The Novel Software Vgc Analyzer. Radiat Prot Dosimetry 2016;169(1-4):54-9. Doi: 10.1093/rpd/ncw035.

52. Precht H., Hansson J., Outzen C., Hogg P., Tingberg A. Radiography Radiographers ' perspectives ' on Visual Grading Analysis as a scienti fi c method to evaluate image quality. Radiography 2019;(xxxx):6-10. Doi: 10.1016/j.radi.2019.06.006.

53. Ludewig E., Richter A., Frame M. Diagnostic imaging - evaluating image quality using visual grading characteristic (VGC) analysis. Vet Res Commun 2010;34(5):473-9. Doi: 10.1007/s11259-010-9413-2.

54. HCPC - health \& care professional council. Radiographers. The Standards of Proficiency for Radiographers. Available from: https://www.hcpc-uk.org/standards/standards-of-proficiency/radiographers/ [accessed August 21, 2019].

55. Sá dos Reis C., Pires-Jorge JA., York H., Flaction L., Johansen S., Maehle S. Curricula, attributes and clinical experiences of radiography programs in four European educational institutions. Radiography 2018;24(3):e61-8. Doi: 10.1016/j.radi.2018.03.002.

56. Manning D., Ethell S., Donovan T., Crawford T. How do radiologists do it? The influence of experience and training on searching for chest nodules. Radiography 2006;12(2):134-42. Doi: 10.1016/j.radi.2005.02.003.

57. Donovan T., Manning DJ., Crawford T. Performance changes in lung nodule detection following perceptual feedback of eye movements. In: Sahiner B, and Manning DJ, editors. Proc. SPIE 6917, Medical Imaging 2008: Image Perception, Observer Performance, and Technology Assessment, 2008, p. 691703.

58. Lowe JM., Brennan PC., Evanoff MG., McEntee MF. Variations in Performance of LCDs Are Still Evident After DICOM Gray-Scale Standard Display Calibration. Am J Roentgenol 2010;195(1):181-7. Doi: 10.2214/AJR.09.2851. 
